\title{
A differentiable sphere theorem for compact Lagrangian submanifolds in complex Euclidean space and complex projective space
}

\author{
Haizhong Li AND XiAnFeng Wang
}

We obtain a new differentiable sphere theorem for compact Lagrangian submanifolds in complex Euclidean space and complex projective space.

\section{Introduction}

The sphere theorem for Riemannian manifolds was firstly studied by Rauch (see [31]) in 1951, since then there have been many excellent works on sphere theorems for Riemannian manifolds and submanifolds (see [1-3, 5-7, 10-15, 23-26, 29, 34, 35], etc.).

Andrews and Baker proved in [2] by the method of mean curvature flow that, for a compact $n$-dimensional submanifold in $\mathbb{R}^{n+p}$, we denote by $S$ the norm square of the second fundamental form and $H$ the mean curvature, if $H \neq 0$ everywhere and

$$
S \leq a n^{2} H^{2}, \text { where } a \leq \frac{4}{3 n}(2 \leq n \leq 4) ; \quad a \leq \frac{1}{n-1}(n \geq 4),
$$

then $M$ is diffeomorphic to $\mathbb{S}^{n}$.

Brendle and Schoen studied (e.g., [10-15]) the convergence theory for Ricci flow and its application to the differentiable sphere theorem, they proved the following result, which is very important in the proof of our main theorem for $n \geq 4$.

Theorem 1.1 [12]. Let $\left(M, g_{0}\right)$ be a compact, locally irreducible Riemannian manifold of dimension $n \geq 4$. Assume that $M \times \mathbb{R}^{2}$ has nonnegative isotropic curvature, i.e.,

$$
R_{1313}+\lambda^{2} R_{1414}+\mu^{2} R_{2323}+\lambda^{2} \mu^{2} R_{2424}-2 \lambda \mu R_{1234} \geq 0
$$


for all orthonormal four-frames $\left\{e_{1}, e_{2}, e_{3}, e_{4}\right\}$ and all $\lambda, \mu \in[-1,1]$. Then one of the following statements holds:

(i) $M$ is diffeomorphic to a spherical space form.

(ii) $n=2 m$ and the universal cover of $M$ is a Kähler manifold biholomorphic to $\mathbb{C} P^{m}$.

(iii) The universal cover of $M$ is isometric to a compact symmetric space.

In [24], by applying Theorem 1.1, Xu and Gu proved that (cf. [37] and $[38])$

Theorem 1.2 [24]. Let $M$ be an $n(\geq 4)$-dimensional oriented complete submanifold in an $N$-dimensional simply connected space form $F^{N}(c)$ with $c \geq 0$. Assume that

$$
S \leq \frac{n^{2} H^{2}}{n-1}+2 c,
$$

where $c+H^{2}>0$. We have

(i) If $c=0$, then $M$ is either diffeomorphic to $S^{n}, \mathbb{R}^{n}$, or locally isometric to $S^{n-1}(r) \times \mathbb{R}$.

(ii) If $M$ is compact, then $M$ is diffeomorphic to $S^{n}$.

Let $\bar{M}^{n}(4 c)$ be a complex space form with constant holomorphic sectional curvature $4 c$. When $c=0, \quad \bar{M}^{n}(4 c)=\mathbb{C}^{n} ; \quad$ when $c>0, \quad \bar{M}^{n}(4 c)=$ $\mathbb{C P}^{n}(4 c)$; when $c<0, \bar{M}^{n}(4 c)=\mathbb{C} \mathbb{H}^{n}(4 c)$.

For any Lagrangian submanifold $M$ in a complex space form $\bar{M}^{n}(4 c)$, the norm square of the second fundamental form and the squared mean curvature satisfy the following inequality:

$$
S \geq \frac{3 n^{2} H^{2}}{n+2},
$$

and the equality holds if and only if $M$ is either a totally geodesic submanfold or one of the Whitney spheres in complex space forms (see [9], [19] and [32] for $c=0$; see $[20,21]$ for $c \neq 0$ ).

The explicit expressions of the Whitney spheres in $\mathbb{C}^{n}$ or in $\mathbb{C P}^{n}$ are the following examples.

Example 1. Whitney sphere in $\mathbb{C}^{n}$ (see [9], [19], [27], [32]). It is defined as the Lagrangian immersion of the unit sphere $\mathbb{S}^{n}$, centered at the origin of 
$\mathbb{R}^{n+1}$, in $\mathbb{C}^{n}$, given by

$$
\phi: \mathbb{S}^{n} \rightarrow \mathbb{C}^{n}: \phi\left(x_{1}, x_{2}, \ldots, x_{n}, x_{n+1}\right)=\frac{1+i x_{n+1}}{1+x_{n+1}^{2}}\left(x_{1}, \ldots, x_{n}\right) .
$$

Example 2. Whitney spheres in $\mathbb{C P}^{n}$ (see [18], [21], [27]). They are a one-parameter family of Lagrangian spheres in $\mathbb{C P}^{n}$, given by

$$
\bar{\phi}_{\theta}: \mathbb{S}^{n} \rightarrow \mathbb{C P}^{n}(4):
$$

$$
\bar{\phi}_{\theta}\left(x_{1}, x_{2}, \ldots, x_{n}, x_{n+1}\right)=\pi \circ\left(\frac{\left(x_{1}, \ldots, x_{n}\right)}{c_{\theta}+i s_{\theta} x_{n+1}} ; \frac{s_{\theta} c_{\theta}\left(1+x_{n+1}^{2}\right)+i x_{n+1}}{c_{\theta}^{2}+s_{\theta}^{2} x_{n+1}^{2}}\right),
$$

where $\theta \geq 0, c_{\theta}=\cosh \theta, s_{\theta}=\sinh \theta$, and $\pi: \mathbb{S}^{2 n+1}(1) \rightarrow \mathbb{C P}^{n}(4)$ is the Hopf fibration.

It is well-known that (see [36]) there are no self-shrinking Lagrangian spheres in $\mathbb{C}^{n}$, if $n>1$. Our aim in this paper is to prove a differentiable sphere theorem with weakly pinched conditions for compact Lagrangian submanifolds in $\mathbb{C}^{n}$ or in $\mathbb{C P}^{n}$, and the theorem is also valid for the Whitney spheres. More precisely, we prove the following result.

Theorem 1.3. Let $M$ be an $n(\geq 3)$-dimensional compact Lagrangian submanifold in a complex space form $\bar{M}^{n}(4 c)(c \geq 0)$. We denote by $S$ the norm square of the second fundamental form and $H$ the mean curvature. Assume that

$$
S \leq \frac{3 n^{2} H^{2}}{n+\frac{3}{2}}+2 c
$$

then $M$ is diffeomorphic to a spherical space form. In particular, if $M$ is simply connected, then $M$ is diffeomorphic to $\mathbb{S}^{n}$.

Remark 1.4. When $c=0$, Castro (see [16]) constructed a one-parameter family of Lagrangian spheres in $\mathbb{C}^{n}$ including the Whitney spheres, defined by $\Phi_{q}: \mathbb{S}^{n} \rightarrow \mathbb{C}^{n}$, where

$$
\Phi_{q}\left(x_{1}, \ldots, x_{n}, x_{n+1}\right)=\frac{2^{1 / q} \mathrm{e}^{i \beta_{q}\left(x_{n+1}\right)}}{\left[\left(1+x_{n+1}\right)^{q}+\left(1-x_{n+1}\right)^{q}\right]^{1 / q}}\left(x_{1}, \ldots, x_{n}\right), q>1
$$

with

$$
\beta_{q}\left(x_{n+1}\right)=\frac{2}{q} \arctan \left(\frac{\left(1+x_{n+1}\right)^{q / 2}-\left(1-x_{n+1}\right)^{q / 2}}{\left(1+x_{n+1}\right)^{q / 2}+\left(1-x_{n+1}\right)^{q / 2}}\right)
$$


Here, each $\Phi_{q}$ satisfies that $S=\frac{\left(3 n+q^{2}+2 q-2\right) n^{2} H^{2}}{(n+q)^{2}}$. We note that if

$$
1<q \leq 2+\frac{3+\sqrt{3\left(2 n^{2}+n-3\right)}}{2 n-3},
$$

then $\Phi_{q}$ satisfies our condition (1.5), and $\Phi_{2}$ is the Whitney sphere.

\section{Preliminaries}

In this section, $M$ will always denote an $n$-dimensional Lagrangian submanifold of $\bar{M}^{n}(4 c)$, which is an $n$-dimensional complex space form with constant holomorphic sectional curvature $4 c$. We denote the Levi-Civita connections on $M, \bar{M}^{n}(4 c)$ and the normal bundle by $\nabla, D$ and $\nabla_{X}^{\perp}$, respectively. The formulas of Gauss and Weingarten are given by (see [17], [22], [27], [28])

$$
D_{X} Y=\nabla_{X} Y+h(X, Y), D_{X} \xi=-A_{\xi} X+\nabla_{X}^{\perp} \xi
$$

where $X$ and $Y$ are tangent vector fields and $\xi$ is a normal vector field on $M$.

The Lagrangian condition implies that

$$
\nabla_{X}^{\perp} J Y=J \nabla_{X} Y, A_{J X} Y=-J h(X, Y)=A_{J Y} X
$$

where $h$ is the second fundamental form and $A$ denotes the shape operator.

The above formulas immediately imply that $\langle h(X, Y), J Z\rangle$ is totally symmetric, i.e.,

$$
\langle h(X, Y), J Z\rangle=\langle h(Y, Z), J X\rangle=\langle h(Z, X), J Y\rangle
$$

for tangent vector fields $X, Y$ and $Z$.

For a Lagrangian submanifold $M$ in $\bar{M}^{n}(4 c)$, an orthonormal frame field

$$
e_{1}, \ldots, e_{n}, e_{1 *}, \ldots, e_{n^{*}}
$$

is called an adapted Lagrangian frame field, if $e_{1}, \ldots, e_{n}$ are orthonormal tangent vector fields and $e_{1^{*}}, \ldots, e_{n^{*}}$ are normal vector fields given by

$$
e_{1^{*}}=J e_{1}, \ldots, e_{n^{*}}=J e_{n}
$$

Their dual frame fields are $\theta_{1}, \ldots, \theta_{n}$, the Levi-Civita connection forms and normal connection forms are $\theta_{i j}$ and $\theta_{i^{*} j^{*}}$, respectively. 
We denote the components of the second fundamental form by $h_{i j}^{k^{*}}$, i.e, $h\left(e_{i}, e_{j}\right)=\sum_{k=1}^{n} h_{i j}^{k^{*}} e_{k^{*}}$, thus (2.3) is equivalent to

$$
h_{i j}^{k^{*}}=h_{k j}^{i^{*}}=h_{i k}^{j^{*}}, \quad 1 \leq i, j, k \leq n
$$

The norm square of the second fundamental form is $S=\sum_{i, j, k}\left(h_{i j}^{k^{*}}\right)^{2}$. The mean curvature vector $\vec{H}$ is defined by $\vec{H}=\frac{1}{n} \sum_{i, k} h_{i i}^{k^{*}} e_{k^{*}}$ and the mean curvature $H=|\vec{H}|$.

If we denote the components of curvature tensor of $\nabla$ by $R_{i j k l}$, then the equations of Gauss are given by (see [17], [22], [27], [28])

$$
R_{i j k l}=c\left(\delta_{i k} \delta_{j l}-\delta_{i l} \delta_{j k}\right)+\sum_{r=1}^{n}\left(h_{i k}^{r^{*}} h_{j l}^{r^{*}}-h_{i l}^{r^{*}} h_{j k}^{r^{*}}\right) .
$$

\section{Some lemmas and the proof of Theorem 1.3}

We need the following lemmas to finish the proof of Theorem 1.3.

In view of a result of Aubin, we have the following lemma:

Lemma 3.1 [4]. Let $M$ be a compact n-dimensional Riemannian manifold. If $M$ has nonnegative Ricci curvature everywhere and has positive Ricci curvature at some point, then $M$ admits a metric with positive Ricci curvature everywhere.

The following convergence result for Ricci flow in three-dimension due to Hamilton is very important in the proof of Theorem 1.3 for $n=3$.

Lemma 3.2 [25]. Let $M$ be a compact three-manifold which admits a Riemannian metric with strictly positive Ricci curvature. Then, $M$ also admits a metric of constant positive curvature.

A Riemannian manifold $M$ is said to have nonnegative (positive, respectively) isotropic curvature, if

$$
R_{1313}+R_{1414}+R_{2323}+R_{2424}-2 R_{1234} \geq 0 \text { (>0, respectively) }
$$

for all orthonormal four-frames $\left\{e_{1}, e_{2}, e_{3}, e_{4}\right\}$. This notation was firstly introduced by Micallef and Moore, where they proved the following sphere theorem. 
Lemma 3.3 [29]. Let $M$ be a compact simply connected n-dimensional Riemannian manifold which has positive isotropic curvature, where $n \geq 4$. Then, $M$ is homeomorphic to a sphere.

In [30], Micallef and Wang proved that

Lemma 3.4 [30]. Let $(M, g)$ be a closed even-dimensional Riemannian manifold. If $g$ has positive isotropic curvature, then $b_{2}(M)=0$.

Later, Seshadri proved that the study of compact manifolds with nonnegative isotropic curvature reduces to the study of manifolds with positive isotropic curvature. In view of Seshadri's result, we have

Lemma 3.5 [33]. Let $M$ be a compact n-dimensional Riemannian manifold. If $M$ has nonnegative isotropic curvature everywhere and has positive isotropic curvature at some point, then $M$ admits a metric with positive isotropic curvature everywhere.

In order to use the convergence results for the Ricci flow by Brendle and Schoen (see Theorem 1.1) to prove Theorem 1.3, we will first prove the following key lemma.

Lemma 3.6. Let $M$ be an $n$-dimensional $(n \geq 4)$ Lagrangian submanifold in a complex space form $\bar{M}^{n}(4 c)$ with $c \geq 0$. Suppose that

$$
S \leq \frac{6 n^{2} H^{2}}{2 n+3}+2 c
$$

then

$$
R_{1313}+\lambda^{2} R_{1414}+\mu^{2} R_{2323}+\lambda^{2} \mu^{2} R_{2424}-2 \lambda \mu R_{1234} \geq 0
$$

for all orthonormal four-frames $\left\{e_{1}, e_{2}, e_{3}, e_{4}\right\}$ and all $\lambda, \mu \in[-1,1]$, i.e., $M \times \mathbb{R}^{2}$ has nonnegative isotropic curvature.

Proof. For any orthonormal four-frame $\left\{e_{1}, e_{2}, e_{3}, e_{4}\right\}$, we extend it to be an orthonormal tangent vector field $\left\{e_{1}, \ldots, e_{n}\right\}$, then we get an adapted Lagrangian frame field $\left\{e_{1}, \ldots, e_{n}, e_{1^{*}}=J e_{1}, \ldots, e_{n^{*}}=J e_{n}\right\}$. 
We define

$$
H_{r}=\frac{1}{n} \sum_{j=1}^{n} h_{j j}^{r^{*}}, \quad \forall 1 \leq r \leq n
$$

By (2.5) and Gauss equation (2.6), we have

$$
\begin{aligned}
& R_{1212}=c+\sum_{r=1}^{n}\left(h_{11}^{r^{*}} h_{22}^{r^{*}}-\left(h_{12}^{r^{*}}\right)^{2}\right) \\
& =c+\frac{1}{2}\left(n^{2} H^{2}-S\right) \\
& -\frac{1}{2} \sum_{r=1}^{n}\left[\left[\sum_{i=1}^{n}\left(h_{i i}^{r^{*}}\right)^{2}+\sum_{i \neq j} h_{i i}^{r^{*}} h_{j j}^{r^{*}}\right]-\left[\sum_{i=1}^{n}\left(h_{i i}^{r^{*}}\right)^{2}+\sum_{i \neq j}\left(h_{i j}^{r^{*}}\right)^{2}\right]\right] \\
& +\sum_{r=1}^{n}\left(h_{11}^{r^{*}} h_{22}^{r^{*}}-\left(h_{12}^{r^{*}}\right)^{2}\right) \\
& =c+\frac{1}{2}\left(n^{2} H^{2}-S\right)-\sum_{r=1}^{n}\left[\sum_{1 \leq i<j \leq n}\left(h_{i i}^{r^{*}} h_{j j}^{r^{*}}-\left(h_{i j}^{r^{*}}\right)^{2}\right)-h_{11}^{r^{*}} h_{22}^{r^{*}}+\left(h_{12}^{r^{*}}\right)^{2}\right] \\
& =c+\frac{1}{2}\left(n^{2} H^{2}-S\right)-\sum_{r=1}^{n}\left[\sum_{j=3}^{n}\left(h_{11}^{r^{*}}+h_{22}^{r^{*}}\right) h_{j j}^{r^{*}}+\sum_{3 \leq i<j \leq n} h_{i i}^{r^{*}} h_{j j}^{r^{*}}\right. \\
& \left.-\sum_{j=3}^{n}\left(h_{1 j}^{r^{*}}\right)^{2}-\sum_{2 \leq i<j \leq n}\left(h_{i j}^{r^{*}}\right)^{2}\right] \\
& =c+\frac{1}{2}\left(n^{2} H^{2}-S\right)-\left[\sum_{r=1}^{n}\left(\sum_{j=3}^{n}\left(h_{11}^{r^{*}}+h_{22}^{r^{*}}\right) h_{j j}^{r^{*}}+\sum_{3 \leq i<j \leq n} h_{i i}^{r^{*}} h_{j j}^{r^{*}}\right)\right. \\
& -\left[\sum_{j=3}^{n}\left(h_{1 j}^{1^{*}}\right)^{2}+\sum_{j=3}^{n}\left(h_{1 j}^{j^{*}}\right)^{2}+\sum_{j=3}^{n}\left(h_{1 j}^{2^{*}}\right)^{2}+2 \sum_{3 \leq i<j \leq n}\left(h_{1 j}^{i^{*}}\right)^{2}\right] \\
& -\left[\sum_{2 \leq i, j \leq n, i \neq j}\left(h_{j j}^{i^{*}}\right)^{2}+\sum_{j \geq 3}\left(h_{2 j}^{1^{*}}\right)^{2}+\sum_{3 \leq i<j \leq n}\left(h_{i j}^{1^{*}}\right)^{2}\right. \\
& \left.\left.+3 \sum_{2 \leq i<j<r \leq n}\left(h_{i j}^{r^{*}}\right)^{2}\right]\right] \text {. }
\end{aligned}
$$


Then by (2.5), we have

$$
\begin{aligned}
R_{1212}= & c+\frac{1}{2}\left(n^{2} H^{2}-S\right)-\left[\sum_{r=1}^{n}\left(\sum_{j=3}^{n}\left(h_{11}^{r^{*}}+h_{22}^{r^{*}}\right) h_{j j}^{r^{*}}+\sum_{3 \leq i<j \leq n} h_{i i}^{r^{*}} h_{j j}^{r^{*}}\right)\right. \\
& -\left[\sum_{j=3}^{n}\left(h_{11}^{j^{*}}\right)^{2}+\sum_{j=3}^{n}\left(h_{j j}^{1^{*}}\right)^{2}+\sum_{j=3}^{n}\left(h_{12}^{j^{*}}\right)^{2}+2 \sum_{3 \leq i<j \leq n}\left(h_{i j}^{1^{*}}\right)^{2}\right] \\
& \left.-\left[\sum_{2 \leq i, j \leq n, i \neq j}\left(h_{j j}^{i^{*}}\right)^{2}+\sum_{j \geq 3}\left(h_{12}^{j^{*}}\right)^{2}+\sum_{3 \leq i<j \leq n}\left(h_{i j}^{1^{*}}\right)^{2}+3 \sum_{2 \leq i<j<r \leq n}\left(h_{i j}^{r^{*}}\right)^{2}\right]\right] \\
= & c+\frac{1}{2}\left(n^{2} H^{2}-S\right)+I I_{1}+I I_{2} \\
& +2 \sum_{j=3}^{n}\left(h_{12}^{j^{*}}\right)^{2}+3 \sum_{3 \leq i<j \leq n}\left(h_{i j}^{1^{*}}\right)^{2}+3 \sum_{2 \leq i<j<r \leq n}\left(h_{i j}^{r^{*}}\right)^{2},
\end{aligned}
$$

where

$$
\begin{aligned}
& I I_{1}=-\sum_{r=1}^{2}\left[\sum_{j=3}^{n}\left(h_{11}^{r^{*}}+h_{22}^{r^{*}}\right) h_{j j}^{r^{*}}+\sum_{3 \leq i<j \leq n} h_{i i}^{r^{*}} h_{j j}^{r^{*}}-\sum_{j=3}^{n}\left(h_{j j}^{r^{*}}\right)^{2}\right], \\
& I I_{2}=-\sum_{r=3}^{n}\left[\sum_{j=3}^{n}\left(h_{11}^{r^{*}}+h_{22}^{r^{*}}\right) h_{j j}^{r^{*}}+\sum_{3 \leq i<j \leq n} h_{i i}^{r^{*}} h_{j j}^{r^{*}}-\sum_{j \neq r}\left(h_{j j}^{r^{*}}\right)^{2}\right] .
\end{aligned}
$$

After a straightforward computation, we can rewrite $I I_{1}$ and $I I_{2}$ as follows (see also [8]) by use of (3.1):

$$
\begin{aligned}
I I_{1}= & \frac{1}{2(n+1)} \sum_{r=1}^{2}\left[\sum_{j=3}^{n}\left(\left(h_{11}^{r^{*}}+h_{22}^{r^{*}}\right)-3 h_{j j}^{r^{*}}\right)^{2}+3 \sum_{3 \leq i<j \leq n}\left(h_{i i}^{r^{*}}-h_{j j}^{r^{*}}\right)^{2}\right. \\
& \left.-(n-2)\left(h_{11}^{r^{*}}+\cdots+h_{n n}^{r^{*}}\right)^{2}\right] \\
= & \frac{1}{2(n+1)} \sum_{r=1}^{2}\left[\sum_{j=3}^{n}\left(\left(h_{11}^{r^{*}}+h_{22}^{r^{*}}\right)-3 h_{j j}^{r^{*}}\right)^{2}+3 \sum_{3 \leq i<j \leq n}\left(h_{i i}^{r^{*}}-h_{j j}^{r^{*}}\right)^{2}\right. \\
& \left.-(n-2) n^{2} H_{r}^{2}\right]
\end{aligned}
$$




$$
\begin{aligned}
= & \sum_{r=1}^{2}\left\{\frac{3}{2(n+1)(2 n+3)} n^{2} H_{r}^{2}-\frac{2 n-3}{2(2 n+3)} n^{2} H_{r}^{2}\right. \\
& \left.+\frac{1}{2(n+1)}\left[\sum_{j=3}^{n}\left(\left(h_{11}^{r^{*}}+h_{22}^{r^{*}}\right)-3 h_{j j}^{r^{*}}\right)^{2}+3 \sum_{3 \leq i<j \leq n}\left(h_{i i}^{r^{*}}-h_{j j}^{r^{*}}\right)^{2}\right]\right\},
\end{aligned}
$$

$$
\begin{aligned}
& I I_{2}=\sum_{r=3}^{n}\left\{-\frac{2 n-3}{2(2 n+3)}\left(h_{11}^{r^{*}}+\cdots+h_{n n}^{r^{*}}\right)^{2}\right. \\
& +\frac{1}{2(2 n+3)}\left[\sum_{j \geq 3, j \neq r}\left(2\left(h_{11}^{r^{*}}+h_{22}^{r^{*}}\right)-3 h_{j j}^{r^{*}}\right)^{2}+(2 n+3)\left(h_{11}^{r^{*}}-h_{22}^{r^{*}}\right)^{2}\right. \\
& +6 \sum_{3 \leq i<j \leq n, i \neq r, j \neq r}\left(h_{i i}^{r^{*}}-h_{j j}^{r^{*}}\right)^{2}+2 \sum_{j \geq 3, j \neq r}\left(h_{r r}^{r^{*}}-3 h_{j j}^{r^{*}}\right)^{2} \\
& \left.\left.+3\left(h_{r r}^{r^{*}}-2\left(h_{11}^{r^{*}}+h_{22}^{r^{*}}\right)\right)^{2}\right]\right\} \\
& =\sum_{r=3}^{n}\left\{-\frac{2 n-3}{2(2 n+3)} n^{2} H_{r}^{2}+\frac{1}{2(2 n+3)}\left[\sum_{j \geq 3, j \neq r}\left(2\left(h_{11}^{r^{*}}+h_{22}^{r^{*}}\right)-3 h_{j j}^{r^{*}}\right)^{2}\right.\right. \\
& +(2 n+3)\left(h_{11}^{r^{*}}-h_{22}^{r^{*}}\right)^{2}+6 \sum_{3 \leq i<j \leq n, i \neq r, j \neq r}\left(h_{i i}^{r^{*}}-h_{j j}^{r^{*}}\right)^{2} \\
& \left.\left.+2 \sum_{j \geq 3, j \neq r}\left(h_{r r}^{r^{*}}-3 h_{j j}^{r^{*}}\right)^{2}+3\left(h_{r r}^{r^{*}}-2\left(h_{11}^{r^{*}}+h_{22}^{r^{*}}\right)\right)^{2}\right]\right\} \text {. }
\end{aligned}
$$

Hence, we get

$$
\begin{aligned}
R_{1212}= & \frac{1}{2}\left(\frac{6 n^{2} H^{2}}{2 n+3}+2 c-S\right)+\frac{3}{2(n+1)(2 n+3)}\left(n^{2} H_{1}^{2}+n^{2} H_{2}^{2}\right) \\
& +2 \sum_{j=3}^{n}\left(h_{12}^{j^{*}}\right)^{2}+3 \sum_{3 \leq i<j \leq n}\left(h_{i j}^{1^{*}}\right)^{2}+3 \sum_{2 \leq i<j<r \leq n}\left(h_{i j}^{r^{*}}\right)^{2} \\
& +\frac{1}{2(n+1)} \sum_{r=1}^{2}\left[\sum_{j=3}^{n}\left(\left(h_{11}^{r^{*}}+h_{22}^{r^{*}}\right)-3 h_{j j}^{r^{*}}\right)^{2}+3 \sum_{3 \leq i<j \leq n}\left(h_{i i}^{r^{*}}-h_{j j}^{r^{*}}\right)^{2}\right]
\end{aligned}
$$




$$
\begin{aligned}
& +\frac{1}{2(2 n+3)} \sum_{r=3}^{n}\left[\sum_{j \geq 3, j \neq r}\left(2\left(h_{11}^{r^{*}}+h_{22}^{r^{*}}\right)-3 h_{j j}^{r^{*}}\right)^{2}\right. \\
& +(2 n+3)\left(h_{11}^{r^{*}}-h_{22}^{r^{*}}\right)^{2}+6 \sum_{3 \leq i<j \leq n, i \neq r, j \neq r}\left(h_{i i}^{r^{*}}-h_{j j}^{r^{*}}\right)^{2} \\
& \left.+2 \sum_{j \geq 3, j \neq r}\left(h_{r r}^{r^{*}}-3 h_{j j}^{r^{*}}\right)^{2}+3\left(h_{r r}^{r^{*}}-2\left(h_{11}^{r^{*}}+h_{22}^{r^{*}}\right)\right)^{2}\right] \\
& \geq \frac{1}{2}\left(\frac{6 n^{2} H^{2}}{2 n+3}+2 c-S\right)+2 \sum_{j=3}^{n}\left(h_{12}^{j^{*}}\right)^{2}+3 \sum_{3 \leq i<j \leq n}\left(h_{i j}^{1^{*}}\right)^{2} \\
& +3 \sum_{2 \leq i<j<r \leq n}\left(h_{i j}^{r^{*}}\right)^{2}+\frac{1}{2} \sum_{r=3}^{n}\left(h_{11}^{r^{*}}-h_{22}^{r^{*}}\right)^{2} \sum_{\geq} \\
& \frac{1}{2}\left(\frac{6 n^{2} H^{2}}{2 n+3}+2 c-S\right),
\end{aligned}
$$

and the equality in the last inequality holds only if the components of the second fundamental form satisfy that

$$
\left\{\begin{array}{l}
h_{11}^{1^{*}}=-h_{22}^{1^{*}}=a_{1}, \quad h_{11}^{2^{*}}=-h_{22}^{2^{*}}=a_{2}, \\
h_{11}^{r^{*}}=h_{22}^{r^{*}}=3 b_{r}, \quad h_{j j}^{r^{*}}=4 b_{r}, \quad h_{r r}^{r^{*}}=12 b_{r}, \quad j, r \geq 3, j \neq r,
\end{array}\right.
$$

for some numbers $a_{1}, a_{2}, b_{r}$, and the other components of the second fundamental form are 0 .

By using the first Bianchi identity, (2.5) and Gauss equation (2.6), we have

$$
\begin{aligned}
-R_{1234}= & R_{1342}+R_{1423} \\
= & \sum_{r=1}^{n}\left(h_{14}^{r^{*}} h_{23}^{r^{*}}-h_{12}^{r^{*}} h_{34}^{r^{*}}+h_{12}^{r^{*}} h_{34}^{r^{*}}-h_{13}^{r^{*}} h_{24}^{r^{*}}\right) \\
= & \sum_{r \geq 5}\left(h_{14}^{r^{*}} h_{23}^{r^{*}}-h_{13}^{r^{*}} h_{24}^{r^{*}}\right)+h_{14}^{1^{*}} h_{23}^{1^{*}}-h_{12}^{1^{*}} h_{34}^{1^{*}}+h_{12}^{1^{*}} h_{34}^{1^{*}}-h_{13}^{1^{*}} h_{24}^{1^{*}} \\
& +h_{14}^{2^{*}} h_{23}^{2^{*}}-h_{12}^{2^{*}} h_{34}^{2^{*}}+h_{12}^{2^{*}} h_{34}^{2^{*}}-h_{13}^{2^{*}} h_{24}^{2^{*}}+h_{14}^{3^{*}} h_{23}^{3^{*}}-h_{12}^{3^{*}} h_{34}^{3^{*}} \\
& +h_{12}^{3^{*}} h_{34}^{3^{*}}-h_{13}^{3^{*}} h_{24}^{3^{*}}+h_{14}^{4^{*}} h_{23}^{4^{*}}-h_{12}^{4^{*}} h_{34}^{4^{*}}+h_{12}^{4^{*}} h_{34}^{4^{*}}-h_{13}^{4^{*}} h_{24}^{4^{*}}
\end{aligned}
$$




$$
\begin{aligned}
= & \sum_{r \geq 5}\left(h_{14}^{r^{*}} h_{23}^{r^{*}}-h_{13}^{r^{*}} h_{24}^{r^{*}}\right)+\left(h_{33}^{2^{*}}-h_{11}^{2^{*}}\right) h_{34}^{1^{*}}+\left(h_{11}^{4^{*}}-h_{33}^{4^{*}}\right) h_{12}^{3^{*}} \\
& +\left(h_{11}^{2^{*}}-h_{44}^{2^{*}}\right) h_{34}^{1^{*}}+\left(h_{44}^{3^{*}}-h_{11}^{3^{*}}\right) h_{12}^{4^{*}}+\left(h_{22}^{1^{*}}-h_{33}^{1^{*}}\right) h_{23}^{4^{*}} \\
& +\left(h_{33}^{4^{*}}-h_{22}^{4^{*}}\right) h_{23}^{1^{*}}+\left(h_{44}^{1^{*}}-h_{22}^{1^{*}}\right) h_{23}^{4^{*}}+\left(h_{22}^{3^{*}}-h_{44}^{3^{*}}\right) h_{12}^{4^{*}},
\end{aligned}
$$

which together with (3.7) immediately give that

$$
\begin{aligned}
& R_{1313}+\lambda^{2} R_{1414}+\mu^{2} R_{2323}+\lambda^{2} \mu^{2} R_{2424}-2 \lambda \mu R_{1234} \\
& \geq \frac{1+\lambda^{2}+\mu^{2}+\lambda^{2} \mu^{2}}{2}\left(\frac{6 n^{2} H^{2}}{2 n+3}+2 c-S\right)+2 \sum_{j \neq 1,3}\left(h_{13}^{j^{*}}\right)^{2} \\
& +3 \sum_{i, j \neq 1,3, i<j}\left(h_{i j}^{1^{*}}\right)^{2}+3 \sum_{i<j<r, i \neq 1}\left(h_{i j}^{r^{*}}\right)^{2}+\frac{1}{2} \sum_{r \neq 1,3}\left(h_{11}^{r^{*}}-h_{33}^{r^{*}}\right)^{2} \\
& +\lambda^{2}\left[2 \sum_{j \neq 1,4}\left(h_{14}^{j^{*}}\right)^{2}+3 \sum_{i, j \neq 1,4, i<j}\left(h_{i j}^{1^{*}}\right)^{2}+3 \sum_{i<j<r, i \neq 1}\left(h_{i j}^{r^{*}}\right)^{2}\right. \\
& \left.+\frac{1}{2} \sum_{r \neq 1,4}\left(h_{11}^{r^{*}}-h_{44}^{r^{*}}\right)^{2}\right]+\mu^{2}\left[2 \sum_{j \neq 2,3}\left(h_{23}^{j^{*}}\right)^{2}+3 \sum_{i, j \neq 2,3, i<j}\left(h_{i j}^{2^{*}}\right)^{2}\right. \\
& \left.+3 \sum_{i<j<r, i \neq 2, j \neq 2}\left(h_{i j}^{r^{*}}\right)^{2}+\frac{1}{2} \sum_{r \neq 2,3}\left(h_{22}^{r^{*}}-h_{33}^{r^{*}}\right)^{2}\right] \\
& +\lambda^{2} \mu^{2}\left[2 \sum_{j \neq 2,4}\left(h_{24}^{j^{*}}\right)^{2}+3 \sum_{i, j \neq 2,4, i<j}\left(h_{i j}^{2^{*}}\right)^{2}\right. \\
& \left.+3 \sum_{i<j<r, i \neq 2, j \neq 2}\left(h_{i j}^{r^{*}}\right)^{2}+\frac{1}{2} \sum_{r \neq 2,4}\left(h_{22}^{r^{*}}-h_{44}^{r^{*}}\right)^{2}\right] \\
& +2 \lambda \mu\left[\sum_{r \geq 5}\left(h_{14}^{r^{*}} h_{23}^{r^{*}}-h_{13}^{r^{*}} h_{24}^{r^{*}}\right)+\left(h_{33}^{2^{*}}-h_{11}^{2^{*}}\right) h_{34}^{1^{*}}+\left(h_{11}^{4^{*}}-h_{33}^{4^{*}}\right) h_{12}^{3^{*}}\right. \\
& +\left(h_{11}^{2^{*}}-h_{44}^{2^{*}}\right) h_{34}^{1^{*}}+\left(h_{44}^{3^{*}}-h_{11}^{3^{*}}\right) h_{12}^{4^{*}}+\left(h_{22}^{1^{*}}-h_{33}^{1^{*}}\right) h_{23}^{4^{*}}+\left(h_{33}^{4^{*}}-h_{22}^{4^{*}}\right) h_{23}^{1^{*}} \\
& \left.+\left(h_{44}^{1^{*}}-h_{22}^{1^{*}}\right) h_{23}^{4^{*}}+\left(h_{22}^{3^{*}}-h_{44}^{3^{*}}\right) h_{12}^{4^{*}}\right] \text {. }
\end{aligned}
$$


By rewriting the right-hand side of (3.10), we have

$$
\begin{aligned}
& R_{1313}+\lambda^{2} R_{1414}+\mu^{2} R_{2323}+\lambda^{2} \mu^{2} R_{2424}-2 \lambda \mu R_{1234} \\
& \geq \frac{1+\lambda^{2}+\mu^{2}+\lambda^{2} \mu^{2}}{2}\left(\frac{6 n^{2} H^{2}}{2 n+3}+2 c-S\right) \\
& +2 \sum_{j \neq 1,3}\left(h_{13}^{j^{*}}\right)^{2}+2 \lambda^{2} \sum_{j \neq 1,4}\left(h_{14}^{j^{*}}\right)^{2}+2 \mu^{2} \sum_{j \neq 2,3}\left(h_{23}^{j^{*}}\right)^{2}+2 \lambda^{2} \mu^{2} \sum_{j \neq 2,4}\left(h_{24}^{j^{*}}\right)^{2} \\
& +2 \lambda \mu \sum_{r \geq 5}\left(h_{14}^{r^{*}} h_{23}^{r^{*}}-h_{13}^{r^{*}} h_{24}^{r^{*}}\right) \\
& +3 \sum_{i, j \neq 1,3, i<j}\left(h_{i j}^{1^{*}}\right)^{2}+3 \sum_{i \neq 1, i<j<r}\left(h_{i j}^{r^{*}}\right)^{2}+\frac{1}{2} \lambda^{2} \mu^{2} \sum_{r \neq 2,4}\left(h_{22}^{r^{*}}-h_{44}^{r^{*}}\right)^{2} \\
& +2 \lambda \mu\left[\left(h_{44}^{1^{*}}-h_{22}^{1^{*}}\right) h_{23}^{4^{*}}+\left(h_{22}^{3^{*}}-h_{44}^{3^{*}}\right) h_{12}^{4^{*}}\right] \\
& +\lambda^{2}\left[3 \sum_{i, j \neq 1,4, i<j}\left(h_{i j}^{1^{*}}\right)^{2}+3 \sum_{i \neq 1, i<j<r}\left(h_{i j}^{r^{*}}\right)^{2}\right]+\frac{1}{2} \mu^{2} \sum_{r \neq 2,3}\left(h_{22}^{r^{*}}-h_{33}^{r^{*}}\right)^{2} \\
& +2 \lambda \mu\left[\left(h_{22}^{1^{*}}-h_{33}^{1^{*}}\right) h_{23}^{4^{*}}+\left(h_{33}^{4^{*}}-h_{22}^{4^{*}}\right) h_{23}^{1^{*}}\right] \\
& +\mu^{2}\left[3 \sum_{i, j \neq 2,3, i<j}\left(h_{i j}^{2^{*}}\right)^{2}+3 \sum_{i \neq 2, j \neq 2, i<j<r}\left(h_{i j}^{r^{*}}\right)^{2}\right]+\frac{1}{2} \lambda^{2} \sum_{r \neq 1,4}\left(h_{11}^{r^{*}}-h_{44}^{r^{*}}\right)^{2} \\
& +2 \lambda \mu\left[\left(h_{11}^{2^{*}}-h_{44}^{2^{*}}\right) h_{34}^{1^{*}}+\left(h_{44}^{3^{*}}-h_{11}^{3^{*}}\right) h_{12}^{4^{*}}\right] \\
& +\lambda^{2} \mu^{2}\left[3 \sum_{i, j \neq 2,4, i<j}\left(h_{i j}^{2^{*}}\right)^{2}+3 \sum_{i \neq 2, j \neq 2, i<j<r}\left(h_{i j}^{r^{*}}\right)^{2}\right]+\frac{1}{2} \sum_{r \neq 1,3}\left(h_{11}^{r^{*}}-h_{33}^{r^{*}}\right)^{2} \\
& +2 \lambda \mu\left[\left(h_{33}^{2^{*}}-h_{11}^{2^{*}}\right) h_{34}^{1^{*}}+\left(h_{11}^{4^{*}}-h_{33}^{4^{*}}\right) h_{12}^{3^{*}}\right] \\
& \geq \frac{1+\lambda^{2}+\mu^{2}+\lambda^{2} \mu^{2}}{2}\left(\frac{6 n^{2} H^{2}}{2 n+3}+2 c-S\right) \text {. }
\end{aligned}
$$

Hence, by the assumption that $S \leq \frac{6 n^{2} H^{2}}{2 n+3}+2 c$, we get

$$
R_{1313}+\lambda^{2} R_{1414}+\mu^{2} R_{2323}+\lambda^{2} \mu^{2} R_{2424}-2 \lambda \mu R_{1234} \geq 0,
$$

i.e., $M \times \mathbb{R}^{2}$ has nonnegative isotropic curvature.

Proof of Theorem 1.3: We have two cases: (i) $c>0$ and (ii) $c=0$. In each case, we will discuss two subcases: $n=3$ and $n \geq 4$. When $n=3$, we note that (3.7) is still true. When $n \geq 4$, we denote by $\tilde{M}$ the universal cover 
of $M$, from Lemma 3.6 we know that $M \times \mathbb{R}^{2}$ has nonnegative isotropic curvature, hence $\tilde{M} \times \mathbb{R}^{2}$ also has nonnegative isotropic curvature.

Case (i) $c>0$.

If $n=3$, for any unit tangent vector $u \in T_{p} M$ at a point $p \in M$, we can choose an orthonormal three-frame $\left\{e_{1}, e_{2}, e_{3}\right\}$ such that $e_{1}=u$. From (3.7) we have

$$
\begin{aligned}
\operatorname{Ric}(u) & =R_{1212}+R_{1313} \\
& \geq \frac{6 n^{2} H^{2}}{2 n+3}+2 c-S=6 H^{2}+2 c-S,
\end{aligned}
$$

and the equality holds only if $R_{1212}=R_{1313}=\frac{1}{2}\left(6 H^{2}+2 c-S\right)$, then from (3.8) we get the equality only if $h_{i j}^{k^{*}}=0,1 \leq i, j, k \leq 3$, which means that $p$ is a totally geodesic point, and hence $S=6 H^{2}$. Hence, we conclude that $M$ has positive Ricci curvature. This together with Hamilton's theorem (see Lemma 3.2) imply that $M$ is diffeomorphic to a spherical space form. In particular, if $M$ is simply connected, then $M$ is diffeomorphic to $\mathbb{S}^{3}$.

If $n \geq 4$, for any unit vector $u$ at a point $p \in M$, take $e_{1}=u$, then we have $\operatorname{Ric}(u)=\sum_{k=2}^{n} R_{1 k 1 k}$, from (3.7) we know that $\operatorname{Ric}(u) \geq \frac{n-1}{2}\left(\frac{6 n^{2} H^{2}}{2 n+3}+2 c-\right.$ $S)$, and the equality holds only if $R_{1212}=\cdots=R_{1 n 1 n}=\frac{1}{2}\left(\frac{6 n^{2} H^{2}}{2 n+3}+2 c-S\right)$, then from (3.8) we get the equality only if $h_{i j}^{k^{*}}=0,1 \leq i, j, k \leq n$, which means that $p$ is a totally geodesic point, and hence $S=\frac{6 n^{2} H^{2}}{2 n+3}$. Hence, we conclude that $M$ has positive Ricci curvature. Since $M$ is compact and has positive Ricci curvature, $\tilde{M}$ is also compact.

For any orthonormal four-frame $\left\{e_{1}, e_{2}, e_{3}, e_{4}\right\}$ at a point $p \in T_{p} M$, let $\lambda=\mu=1$ in (3.11), we immediately get

$$
R_{1313}+R_{1414}+R_{2323}+R_{2424}-2 R_{1234} \geq 2\left(\frac{6 n^{2} H^{2}}{2 n+3}+2 c-S\right)
$$

and from (3.7), (3.8) and (3.11) we know that the equality in (3.14) holds only if $h_{i j}^{k^{*}}=0,1 \leq i, j, k \leq n$, which means that $p$ is a totally geodesic point, and hence $S=\frac{6 n^{2} H^{2}}{2 n+3}$. Hence, we conclude that $M$ has positive isotropic curvature, which implies that $\tilde{M}$ also has positive isotropic curvature.

We have shown that $\tilde{M}$ is compact and has positive isotropic curvature, by using a theorem due to Micallef and Moore (see Lemma 3.3), we get that $\tilde{M}$ is homeomorphic to $\mathbb{S}^{n}$, hence, $\tilde{M}$ is locally irreducible and the locally symmetric metric of $\tilde{M}$ would have to be of constant positive sectional curvature (see also Remark (ii) on page 622 of [33] and Lemma 4 of [24]). As $\tilde{M}$ has positive isotropic curvature, from a theorem of Micallef and Wang (see 
Lemma 3.4), $\tilde{M}$ cannot be a Kähler manifold. Since $\tilde{M} \times \mathbb{R}^{2}$ has nonnegative isotropic curvature, by combining Theorem 1.1 , we conclude that $\tilde{M}$ is diffeomorphic to $\mathbb{S}^{n}$, which implies that $M$ is diffeomorphic to a spherical space form. In particular, if $M$ is simply connected, then $M$ is diffeomorphic to $\mathbb{S}^{n}$.

Case (ii) $c=0$.

If $n=3$, for any unit tangent vector $u \in T_{p} M$ at the point $p \in M$, we can choose an orthonormal three-frame $\left\{e_{1}, e_{2}, e_{3}\right\}$ such that $e_{1}=u$. From (3.7) we have

$$
\begin{aligned}
\operatorname{Ric}(u) & =R_{1212}+R_{1313} \\
& \geq \frac{6 n^{2} H^{2}}{2 n+3}-S=6 H^{2}-S,
\end{aligned}
$$

and the equality holds only if $R_{1212}=R_{1313}=\frac{1}{2}\left(\frac{6 n^{2} H^{2}}{2 n+3}+2 c-S\right)$, then from (3.8) we get the equality only if $h_{i j}^{k^{*}}=0,1 \leq i, j, k \leq 3$, which means that $p$ is a totally geodesic point, and hence $S=6 H^{2}$. Hence, we get that $\operatorname{Ric}(u) \geq 0$ and $\operatorname{Ric}(u)=0$ can only happen at the totally geodesic points. Since $M$ is a compact submanifold in $\mathbb{C}^{n}, M$ cannot be minimal and hence can not be totally geodesic, which implies that there exists a point $p \in M$ such that $\operatorname{Ric}(u)>0$ for any unit tangent vector $u \in T_{p} M$, i.e., we get that the Ricci curvature of $M$ is quasi-positive, then by Aubin's theorem (see Lemma 3.1) $M$ admits a metric with positive Ricci curvature. This together with Hamilton's theorem (see Lemma 3.2) imply that $M$ is diffeomorphic to a spherical space form. In particular, if $M$ is simply connected, then $M$ is diffeomorphic to $\mathbb{S}^{3}$.

If $n \geq 4$, after a similar argument to that in the case $n=3$, we get that the Ricci curvature of $M$ is quasi-positive, then by Aubin's theorem (see Lemma 3.1) $M$ admits a metric with positive Ricci curvature. Since $M$ is compact and has positive Ricci curvature, $\tilde{M}$ is also compact.

For any orthonormal four-frame $\left\{e_{1}, e_{2}, e_{3}, e_{4}\right\}$ at a point $p \in T_{p} M$, let $\lambda=\mu=1$ in (3.11), we immediately get

$$
R_{1313}+R_{1414}+R_{2323}+R_{2424}-2 R_{1234} \geq 2\left(\frac{6 n^{2} H^{2}}{2 n+3}-S\right),
$$

from (3.7), (3.8) and (3.11) we know that the equality in (3.16) holds only if $h_{i j}^{k^{*}}=0,1 \leq i, j, k \leq n$, which means that $p$ is a totally geodesic point, and hence $S=\frac{6 n^{2} H^{2}}{2 n+3}$. We conclude that $M$ has nonnegative isotropic curvature and has positive isotropic curvature for some point in $M$, which together 
with Lemma 3.5 imply that $M$ admits a metric with positive isotropic curvature. Therefore, $\tilde{M}$ also admits a metric with positive isotropic curvature.

We have shown that $\tilde{M}$ is compact and admits a metric with positive isotropic curvature, by using a theorem due to Micallef and Moore (see Lemma 3.3), we get that $\tilde{M}$ is homeomorphic to $\mathbb{S}^{n}$, hence $\tilde{M}$ is locally irreducible and the locally symmetric metric of $\tilde{M}$ would have to be of constant positive sectional curvature (see also Remark (ii) on page 622 of [33] and Lemma 4 of [24]). As $\tilde{M}$ admits a metric with positive isotropic curvature, from a theorem of Micallef and Wang (see Lemma 3.4), $\tilde{M}$ cannot be a Kähler manifold. Since $\tilde{M} \times \mathbb{R}^{2}$ has nonnegative isotropic curvature, by combining Theorem 1.1, we conclude that $\tilde{M}$ is diffeomorphic to $\mathbb{S}^{n}$, which implies that $M$ is diffeomorphic to a spherical space form. In particular, if $M$ is simply connected, then $M$ is diffeomorphic to $\mathbb{S}^{n}$.

This completes the proof of Theorem 1.3.

\section{A Differentiable sphere theorem for compact Lagrangian submanifolds in a Kähler manifold}

In this section, we extend Theorem 1.3 to compact Lagrangian submanifolds in a Kähler manifold. We have the following theorem.

Theorem 4.1. Let $M$ be an $n(\geq 3)$-dimensional compact Lagrangian submanifold in a Kähler manifold $\bar{M}^{n}$. We denote by $S$ the norm square of the second fundamental form and $H$ the mean curvature. Let $\bar{K}(u \wedge v)$ denote the sectional curvature of the 2-dimensional subspace of $T_{p} \bar{M}^{n}$ spanned by $u$ and $v$. Assume that

$$
S \leq \frac{3 n^{2} H^{2}}{n+\frac{3}{2}}+\frac{2}{3}(4 \delta-\Delta)
$$

where $\quad \Delta=\max _{u, v \in T_{p} \bar{M}^{n},\langle u, J v\rangle=0} \bar{K}(u \wedge v), \quad \delta=\min _{u, v \in T_{p} \bar{M}^{n},\langle u, J v\rangle=0} \bar{K}$ $(u \wedge v)$. If $4 \delta-\Delta \equiv 0$ on $\bar{M}^{n}$, we assume moreover that $M$ is not totally geodesic. Then, $M$ is diffeomorphic to a spherical space form. In particular, if $M$ is simply connected, then $M$ is diffeomorphic to $\mathbb{S}^{n}$.

Proof. Let $M$ be a Lagrangian submanifold in a Kähler manifold $\bar{M}^{n}$, if we denote the Levi-Civita connections on $M, \bar{M}^{n}$ and the normal bundle by $\nabla, D$ and $\nabla_{X}^{\perp}$, respectively, then $(2.1)-(2.5)$ still hold. 
If we denote the components of curvature tensor of $\nabla$ and $D$ by $R_{i j k l}$ and $\bar{R}_{i j k l}$, respectively, then the equations of Gauss are given by

$$
R_{i j k l}=\bar{R}_{i j k l}+\sum_{r=1}^{n}\left(h_{i k}^{r^{*}} h_{j l}^{r^{*}}-h_{i l}^{r^{*}} h_{j k}^{r^{*}}\right) .
$$

By a similar method of proving Berger's inequality (see [6], or see [3], Lemma 2.50), for orthonormal frames $\left\{e_{i}, e_{j}, e_{k}, e_{l}\right\}$ which are orthogonal to $\left\{J e_{i}, J e_{j}, J e_{k}, J e_{l}\right\}$, we have

$$
\left|\bar{R}_{i j k l}\right| \leq \frac{2}{3}(\Delta-\delta)
$$

By using (4.2) and (4.3) together instead of (2.6), Theorem 4.1 can be proved after an argument analogous to that in the proof of Theorem 1.3, we omit the details here.

Remark 4.2. We note that Theorem 4.1 is a generalization of Theorem 1.3. In Theorem 4.1 , if we take $\bar{M}^{n}$ to be a complex space form $\bar{M}^{n}(4 c)$ with $c \geq 0$, then we immediately get Theorem 1.3 .

\section{Acknowledgments}

The authors would like to express their thanks to Professor Ben Andrews for his helpful discussion and valuable suggestions. The authors would like to express their thanks to the referees for their valuable comments and suggestions. The first author was supported in part by NSFC grant no. 11271214. The second author was supported in part by NSFC grant no. 11171175, NSFC grant no. 11201243, "Specialized Research Fund for the Doctoral Program of Higher Education, grant no. 20120031120026" and "the Fundamental Research Funds for the Central Universities".

\section{References}

[1] B. Andrews, Positively curved surfaces in the three-sphere, in Proc. Int. Congress of Mathematicians, vol. II, Beijing, 2002) (Beijing), Higher Ed. Press, 2002, pp. 221-230.

[2] B. Andrews and C. Baker, Mean curvature flow of pinched submanifolds to spheres, J. Differ. Geom. 85(3) (2010), 357-395. 
[3] B. Andrews and C. Hopper, The Ricci flow in Riemannian geometry: complete proof of the differentiable 1/4-pinching sphere theorem, Lecture Notes in Mathematics, vol. 2011, Springer, Heidelberg, 2011.

[4] T. Aubin, Métriques riemanniennes et courbure, J. Differ. Geom. 4 (1970), 383-424.

[5] M. Berger, Les variétés Riemanniennes (1/4)-pincées, Ann. Scuola Normale Super Pisa (3) 14(1960), 161-170.

[6] M. Berger, Riemannian geometry during the second half of the twentieth century, University Lecture Series, (11), American Mathematical Society, Providence, RI, 2000, Reprint of the 1998 original.

[7] C. Böhm and B. Wilking, Manifolds with positive curvature operators are space forms, Ann. Math. (2) 167(3) (2008), 1079-1097.

[8] J. Bolton, F. Dillen, J. Fastenakels and Luc Vrancken, A best possible inequality for curvature-like tensor fields, Math. Inequalities Appl. $\mathbf{1 2}(3)$ (2009), 663-681.

[9] V. Borrelli, B.Y. Chen and J.M. Morvan, Une caractérisation géométrique de la sphère de Whitney, C. R. Acad. Sci. Paris Sér. I Math. 321(11) (1995), 1485-1490.

[10] S. Brendle, A general convergence result for the Ricci flow in higher dimensions, Duke Math. J. 145(3) (2008), 585-601.

[11] S. Brendle, Ricci flow and the sphere theorem, Graduate Studies in Mathematics, 111, American Mathematical Society, Providence, RI, 2010.

[12] S. Brendle and R. Schoen, Classification of manifolds with weakly 1/4pinched curvatures, Acta Math. 200 (2008), no. 1, 1-13.

[13] S. Brendle and R. Schoen,Manifolds with 1/4-pinched curvature are space forms, J. Am. Math. Soc. 22(1) (2009), 287-307.

[14] S. Brendle and R. Schoen, Sphere theorems in geometry, Surveys in differential geometry. Vol. XIII. Geometry, analysis, and algebraic geometry: forty years of the J. Differ. Geom., Surv. Differ. Geom., 13, Int. Press, Somerville, MA, 2009, pp. 49-84.

[15] S Brendle and R. Schoen, Riemannian manifolds of positive curvature, in Proc. Int. Cong. Math. vol. I (New Delhi), Hindustan Book Agency, 2010, pp. 449-475. 
[16] I. Castro, Lagrangian spheres in the complex Euclidean space satisfying a geometric equality, Geom. Dedicata 70(2), (1998), 197-208.

[17] I. Castro, H. Li and F. Urbano, Hamiltonian-minimal Lagrangian submanifolds in complex space forms, Pac. J. Math. 227(1) (2006), 43-63.

[18] I. Castro, C.R. Montealegre and F. Urbano, Closed conformal vector fields and Lagrangian submanifolds in complex space forms, Pac. J. Math. 199(2) (2001), 269-302.

[19] I. Castro and F. Urbano, Lagrangian surfaces in the complex Euclidean plane with conformal Maslov form, Tohoku Math. J. (2) 45 (1993), no. $4,565-582$.

[20] B.Y. Chen, Jacobi's elliptic functions and Lagrangian immersions, Proc. R. Soc. Edinb. Sect. A 126(4) (1996), 687-704.

[21] B.Y. Chen and L. Vrancken, Lagrangian submanifolds satisfying a basic equality, Math. Proc. Camb. Phil. Soc. 120(2) (1996), 291-307.

[22] F. Dillen, H. Li, L. Vrancken and X. Wang, Lagrangian submanifolds in complex projective space with parallel second fundamental form, Pac. J. Math. 255(1) (2012), 79-115.

[23] K. Grove and K. Shiohama, A generalized sphere theorem, Ann. Math. (2) 106(2) (1977), 201-211.

[24] J.R. Gu and H.W. Xu, The sphere theorems for manifolds with positive scalar curvature, J. Differ. Geom. 92(3) (2012), 507-545.

[25] R.S. Hamilton, Three-manifolds with positive Ricci curvature, J. Differ. Geom. 17(2) (1982), 255-306.

[26] W. Klingenberg, Über Riemannsche Mannigfaltigkeiten mit positiver Krümmung, Comment. Math. Helv. 35 (1961), 47-54.

[27] H. Li and L. Vrancken, A basic inequality and new characterization of Whitney spheres in a complex space form, Israel J. Math. 146 (2005), 223-242.

[28] H. Li and X. Wang, Isotropic Lagrangian submanifolds in complex Euclidean space and complex hyperbolic space, Results Math. 56(1-4) (2009), 387-403. 
[29] M.J. Micallef and J.D. Moore, Minimal two-spheres and the topology of manifolds with positive curvature on totally isotropic two-planes, Ann. Math. (2) 127(1) (1988), 199-227.

[30] M.J. Micallef and M.Y. Wang, Metrics with nonnegative isotropic curvature, Duke Math. J. 72(3) (1993), 649-672.

[31] H.E. Rauch, A contribution to differential geometry in the large, Ann. Math. (2) 54 (1951), 38-55.

[32] A. Ros and F. Urbano, Lagrangian submanifolds of $\mathbf{C}^{n}$ with conformal Maslov form and the Whitney sphere, J. Math. Soc. Japan 50 (1998), no. 1, 203-226.

[33] H. Seshadri, Manifolds with nonnegative isotropic curvature, Comm. Anal. Geom. 17(4) (2009), 621-635.

[34] K. Shiohama, Recent developments in sphere theorems, Differential geometry: Riemannian geometry (Los Angeles, CA, 1990), in Proc. Symp. Pure Mathematics, 54, American Mathematical Society, Providence, RI, 1993, pp. 551-576.

[35] K. Shiohama, Sphere theorems, Handbook of differential geometry, Vol. I, North-Holland, Amsterdam, 2000, pp. 865-903.

[36] K. Smoczyk, Mean curvature flow in higher codimension: Introduction and survey, Global Differential Geometry, Springer, Berlin, Heidelberg, 2012, pp. 231-274.

[37] H.W. Xu and J.R. Gu, An optimal differentiable sphere theorem for complete manifolds, Math. Res. Lett. 17(6) (2010), 1111-1124.

[38] H. W. Xu and E. T. Zhao, Topological and differentiable sphere theorems for complete submanifolds, Comm. Anal. Geom. 17(3) (2009), 565-585. 
${ }^{1}$ Department of Mathematical Sciences and Mathematical Sciences Center TSINGHUA UNIVERSITY

BEIJING 100084

People's Republic of ChinA

Email address: hli@math.tsinghua.edu.cn

${ }^{2}$ School of Mathematical Sciences and LPMC NANKAI UNIVERSITY

TiANJin 300071

People's Republic of China

Email address: wangxianfeng@nankai.edu.cn

Received September 7, 2011 